\title{
Verità e giustificazione nel processo penale ${ }^{1}$
}

\author{
Truth and justification in the criminal procedure
}

Verdade e justificação no processo penal

Francesco Caprioli

Professore Ordinario di Procedura penale

Università degli Studi di Torino/Itália

francesco.caprioli@unito.it

http://orcid.org/0000-0003-4038-809X

\begin{abstract}
RIAssunto: Dopo avere premesso che l'unica verità che il giudice penale è tenuto a dichiarare è la "triste verità" della colpevolezza dell'imputato, l'Autore di questo articolo contesta la diffusa opinione che si tratti di una verità minore, formale, convenzionale, non assimilabile alla verità dello scienziato o dello storico. Anche nel processo penale si persegue la Verità con la $\mathrm{V}$ maiuscola, da intendersi come corrispondenza dell'enunciato d'accusa ai fatti in esso descritti. Solo a queste condizioni, del resto, la sentenza di condanna può ritenersi razionalmente giustificata.
\end{abstract}

PARole-Chiave: Processo penale; verità; valutazione della prova; processo penale italiano

AвSTRACT: After clarifying that the only truth that criminal judge must declare is the "sad truth" of the defendant's culpability, the Author of this article disputes the widespread opinion that this truth would be a minor and conventional truth, not comparable to scientific or historical ones. Also in the criminal proceedings we pursue the Truth with capital T, which must be regarded as correspondence

1 Testo della relazione svolta nell'ambito del Convegno "Verità del precetto e della sanzione penale alla prova del processo", Milano, Università Cattolica del Sacro Cuore, 22 giugno 2012. Originalmente publicado em: CAPRIOLI, Francesco. Verità e giustificazione nel processo penale. Rivista italiana di diritto e procedura penale, 2013, fasc. 2, p. 608-625. 
between the indictment and the facts described in it. Only under these conditions the judgment of conviction can be considered rationally justified.

KeY-words: Criminal Procedure; Truth; Evidence Valuation; Italian criminal procedure.

Resumo: Depois de esclarecer que a única verdade que o juiz criminal deve declarar é a "triste verdade" da culpabilidade do acusado, o Autor deste artigo questiona a visão disseminada de que essa verdade seria uma verdade menor, formal e convencional, não comparável com a verdade científica ou histórica. Mesmo no processo penal se persegue a Verdade com $V$ maiúsculo, que deve ser entendida como a correspondência do enunciado da acusação e os fatos nela descritos. Somente se atendidas tais condições é que a sentença condenatória poderá ser aceita como racionalmente justificada.

Palavras-chave: Processo Penal; Verdade; Valoração da prova; Processo penal italiano.

1. Sul tema dei rapporti tra verità e processo circola in ambito penalistico un insidioso luogo comune. Scopo del processo penale sarebbe senz'altro l'accertamento della verità: ma la verità processuale sarebbe una verità minore, formale, convenzionale, da scrivere con l'iniziale minuscola. Costretto dalla logica del ragionamento induttivo a esprimersi in termini meramente probabilistici, e vincolato al rispetto di regole probatorie e criteri normativi di giudizio non sempre funzionali alla ricerca del vero, il giudice penale non potrebbe mai attingere alla verità assoluta, oggettiva, alla Verità con l'iniziale maiuscola - alla verità vera, la verità materiale, la verità storica, la verità dei fatti e via aggettivando e specificando.

Quella che si persegue nel processo penale - è stato scritto «non pretende di essere la verità. [Essa è] più ridotta, quanto al contenuto informativo, di qualunque ipotetica verità 'sostanziale', [sia perché] deve essere suffragata da prove raccolte attraverso tecniche prestabilite normativamente, [sia perché] è sempre una verità solamente probabile e opinabile» ${ }^{2}$. Alla verità processuale non potrebbe che riconoscersi

2 L. Ferrajoli, Diritto e ragione. Teoria del garantismo penale, Laterza, 1991, p. 17. L'idea che il processo penale possa attingere alla verità "materiale" vie- 
«un carattere essenzialmente pratico, non logico-conoscitivo», in quanto il giudice è «sottoposto a limiti [di tempo e di prova] che lo storico ignora», e l'accertamento giudiziale «non sempre è condotto con tutti i mezzi materialmente disponibili» ${ }^{3}$ (né, si potrebbe aggiungere, con tutti i mezzi razionalmente disponibili).

La diffidenza dei processualisti penali nei confronti della verità ha inoltre ben note radici storiche e ideologiche. Non è un caso che nel vigente codice di procedura penale le disposizioni dedicate alla formazione e all'espressione del convincimento giudiziale evitino accuratamente di evocare le categorie del vero e del falso ${ }^{4}$ : e non è un caso che nell'attuale dettato codicistico manchi una norma analoga a quelle contenute nell'art. 299 del codice del 1930, nell'art. 190 comma 1 del codice del 1913 e nell'art. 84 comma 2 del codice del 1865, che invitavano il giudice a compiere tutti gli atti necessari all'accertamento della verità. A guidare il legislatore del 1988 nelle sue scelte lessicali è stata la volontà di cancellare dall'orizzonte normativo un concetto rivelatosi storicamente pernicioso per la legalità processuale ${ }^{5}$. Come ha recentemente ribadito uno dei padri della riforma, si è voluto esorcizzare «un

ne spesso rigettata - in quanto «chimerica», «ingenua», «fuorviante» $[\mathrm{R}$. E. Kostoris, voce Giudizio (dir. proc. pen.), in Enc. giur. Treccani, vol. XV, Agg. 1997, p. 8 s.], figlia di una «concezione epistemologica ormai superata» (D. Negri, Fumus commissi delicti. La prova per le fattispecie cautelari, Giappichelli, 2004, p. 255 s.) - con argomenti che tendono a sovrapporre la verità alla certezza. Sul punto v. infra, § 5 .

3 R. Orlandi, Verità, responsabilità e ravvedimento tra processo penale e pratiche di mediazione, in Corte Assise, 2011, p. 536 s.

4 Significativo, ad esempio, il divieto imposto al giudice, a norma dell'art. 192 comma 2 c.p.p., di «desumere l'esistenza di un fatto» da indizi che non siano gravi, precisi e concordanti. Pochi e di scarso rilievo i richiami alla verità contenuti in altre norme del codice di procedura penale: nell'affermare che il testimone ha l'obbligo di dire la verità e di rispondere secondo verità (artt. 198 comma 1 c.p.p., 497 comma 2 c.p.p.), e nel prescrivere che l'interprete e il perito devono essere avvertiti che il loro incarico non ha altro scopo che quello di far conoscere la verità (artt. 146 comma 2 c.p.p., 226 comma 1 c.p.p.), il legislatore sembra riferirsi esclusivamente al dovere di sincerità che incombe su tali soggetti.

5 Sulle distorsioni del principio di ricerca della verità materiale e del principio del libero convincimento, e sulla loro progressiva metamorfosi in un più generale canone di insofferenza della prova penale a limiti legali non solo nella fase della sua valutazione, ma anche in quella della sua ammissione e della sua 
passato inquisitorio in cui il principio di 'ricerca della verità materiale', in tutto il continente europeo, [era stato] il pilastro di un sistema probatorio che [aveva] consentito di scavalcare qualsiasi ordine o limite nell'iter di accertamento del reato» ${ }^{6}$.

Analoghe tendenze elusive si registrano infine nella giurisprudenza della Corte costituzionale. A inizio anni novanta l'opinione del giudice delle leggi era che «fine primario e ineludibile del processo non [potesse] che essere la ricerca della verità $»^{7}$. Ma in seguito la Corte è sembrata ripiegare su perimetrazioni meno ambiziose dei compiti cognitivi assegnati al giudice ${ }^{8}$ : scopo del processo sarebbe «l'accertamento giudiziale dei fatti di reato e delle relative responsabilità»? .

Come cercheremo di dimostrare, sono cautele ingiustificate e nocive.

2. Conviene immediatamente precisare che nelle pagine che seguono ci si occuperà soltanto della verità dei giudizi di fatto e non

acquisizione, rimane fondamentale l'analisi di M. Nobili, Il principio del libero convincimento del giudice, Giuffré, Milano, 1974, p. 41 ss.

6 E. Amodio, Verso una storia della giustizia penale in età moderna e contemporanea, in Criminalia, 2010, p. 13. Sul punto v. anche la Relazione al Progetto preliminare del codice di procedura penale, Roma, 1988, p. 124 s., in cui il tradizionale richiamo normativo all'accertamento della verità viene ritenuto «frutto di un'iperbole».

7 Così (pur traendone conseguenze assai discutibili) Corte cost. 3 giugno 1992, n. 255: Corte cost. 24 marzo 1993, n. 111; Corte cost. 22 luglio 1994, n. 330. Per Corte cost. 23 maggio 1991, n. 258, anche dopo la riforma del 1988 il processo penale risulterebbe «ispirato al principio di ricerca della verità materiale»; secondo Corte cost. 10 febbraio 1993, n. 41, l'udienza preliminare, a differenza del dibattimento, non sarebbe «una sede di acquisizione probatoria destinata all'accertamento della verità» (così anche Corte cost. 17 giugno 1991, n. 303; Corte cost. 22 maggio 1991, n. 252; Corte cost. 28 gennaio 1991, n. 64).

8 Il riferimento alla verità come «fine primario e ineludibile del processo penale» è invece sopravvissuto nella giurisprudenza di legittimità: cfr. per tutte Cass. 8 febbraio 2005, Cacciatori, in C.e.d. Cass., 231726.

9 Così Corte cost. 2 novembre 1998, n. 361, § 2.1. (v. anche $\S 4.2$. : «la funzione essenziale del processo é quella di accertare e di ricostruire i [fatti], verificare la sussistenza dei reati oggetto del giudizio e di accertare le relative responsabilità»). Sul carattere solo apparentemente meno ambizioso di un simile compito v. infra, § 5. Un cenno alla «ricerca della verità» come scopo della fase dibattimentale ritorna, da ultimo, in Corte cost. 11 marzo 2009, n. 75. 
anche della verità dei giudizi di valore giuridico ${ }^{10}$. Si darà inoltre per scontato che i fatti - compresi quelli di cui si discute nei processi penali - esistono a prescindere dalle nostre interpretazioni e dagli schemi concettuali nei quali fatalmente li inquadriamo. Non si parlerà dunque di costruttivismo e di relativismo in ordine ai fatti, se non per rinviare alle convincenti obiezioni che simili teorie hanno dovuto fronteggiare nel più recente dibattito filosofico ${ }^{11} \mathrm{e}_{\text {giuridico }}{ }^{12}$.

Ciò premesso, occorre innanzitutto stabilire entro quali limiti il giudice penale è chiamato - e si impegna - a 'dire la verità' sui fatti oggetto dell'imputazione.

La risposta è nella struttura stessa del verdetto di colpevolezza, e, prima ancora, nel rapporto che la legge processuale istituisce tra imputazione e sentenza. Dichiarando l'imputato responsabile, il giudice non fa altro che affermare la verità dell'enunciato fattuale contenuto

10 Secondo P. Ferrua, Il giudizio penale: fatto e valore giuridico, in AA.VV., La prova nel dibattimento penale, Giappichelli, 2010, p. 325 s., anche il giudizio di valore giuridico, entro certi limiti, «esprime un'attività conoscitiva, [e dunque può] dirsi vero o falso» (ivi, p. 337). Sarebbe un errore, in particolare, riconoscere natura cognitiva ai soli giudizi di fatto e non anche ai giudizi di diritto, consistendo gli uni e gli altri in una scelta razionalmente giustificata tra diverse plausibili opzioni descrittive (rectius, ri-costruttive) dell'entità fattuale o linguistica che ne costituisce l'oggetto. "Giacché la natura 'cognitiva' del giudizio storico non implica la metafisica pretesa di ricostruire la Realtà-com'è-in-sé e sta soltanto a indicare la possibilità di una giustificazione razionale, di una decisione coerente con le prove (che, per quanto solide, restano logicamente compatibili con una diversa ricostruzione dei fatti), non c'è motivo di ritenere 'anticognitivo' il giudizio di diritto. Più fallibilisti sui fatti, si può essere meno scettici sulle norme» (ivi, p. 336). Sul punto, in diversa prospettiva, M. Taruffo, Contro la veriphobia: osservazioni sparse in risposta a Bruno Cavallone, in Riv. dir. proc., 2010, p. 1006 (nonché Id., La semplice verità, Laterza, 2009, p. 115, e Id., Fatto, prova e verità, in Criminalia, 2009, p. 314), secondo cui «tra le condizioni occorrenti perché una decisione sia formulata secondo diritto» rientrerebbe anche «l'accertamento veritiero dei fatti della causa», ma il giudizio di diritto, in sé e per sé considerato, «non mir[erebbe] a stabilire la verità di alcunché».

11 V. in particolare P.A. Boghossian, Paura di conoscere. Contro il relativismo e il costruttivismo, Carocci, 2006, p. 43 s., 61 s., e D. Marconi, Per la verità. Relativismo e filosofia, Einaudi, 2007, p. 57 s., 69 s., nonché Aa.Vv., Bentornata realtà. Il nuovo realismo in discussione, a cura di M. De Caro e M. Ferraris, Einaudi, 2012, passim.

12 Per tutti M. Taruffo, La semplice verità, cit., p. 74 s. 
nell'imputazione, o, per meglio dire, della proposizione che di quell'enunciato costituisce il contenuto astratto ${ }^{13}$ («poiché le prove dimostrano oltre ogni ragionevole dubbio che Tizio ha ucciso Caio, l'enunciato accusatorio 'Tizio ha ucciso Caio' è vero» ${ }^{14}$ ): il rapporto tra imputazione e condanna riproduce plasticamente l'intuizione tarskiana secondo cui il termine 'vero' collega un linguaggio e un metalinguaggio anziché il linguaggio e la realtà ${ }^{15}$. Perché l'imputato sia assolto, non occorre, invece, che i fatti posti a fondamento dell'accusa si siano rivelati inesistenti, né che sia emersa la verità delle proposizioni corrispondenti alle formule legali di cui all'art. 530 comma 1 c.p.p. Nel dichiarare che 'il fatto non sussiste' o che l'imputato 'non lo ha commesso', il giudice è autorizzato dalla legge ad argomentare ad ignorantiam ${ }^{16}$, cioè, in definitiva, a men-

13 Così impostata, la questione implica «l'idea che i portatori primari di verità siano $[. .$.$] le proposizioni», e che dunque entità come gli enunciati, i proferi-$ menti, le iscrizioni, le affermazioni, le asserzioni, le dichiarazioni - ma anche stati mentali come le credenze, i giudizi, le opinioni, i convincimenti - «siano suscettibili di essere veri o falsi soltanto in un senso secondario e derivato»: così G. Volpe, Teorie della verità, Guerini Scientifica, 2005, p. 68, che definisce tale idea come «la soluzione più classica» del problema di che cosa può essere descritto in termini di verità e falsità (per analoghe conclusioni F. D'Agostini, Introduzione alla verità, Bollati Boringhieri, 2011, p. 42 s.). Di certo - come rileva esattamente $\mathrm{G}$. Ubertis, Conoscenza fattuale e razionalità della decisione giudiziale, in Argomenti di procedura penale, Giuffré, 2002, p. 82 - non sono i fatti a poter essere predicati come veri o falsi, ma solo le proposizioni che li descrivono. Incorre nell'equivoco l'art. 395 comma 4 c.p.c., che allude a «fatti la cui verità è incontrastabilmente esclusa» o "positivamente stabilita».

14 Il giudice penale si pronuncia su una proposizione (e non su un fatto) anche quando condanna Smith perché è un ladro, non solo quando condanna Jones per averlo detto: il riferimento è all'esempio di J. L. Austin riportato in F. D’Agostini, Introduzione alla verità, cit., p. 79 s.

15 Cfr. A. Tarski, La concezione semantica della verità e i fondamenti della semantica, in AA.VV., Semantica e filosofia del linguaggio, a cura di L. Linsky, Il Saggiatore, 1969, p. 31, per il quale, come è noto, "X è vero se e solo se p", dove "p" è «un enunciato qualunque»e " $X$ " è «il nome di questo enunciato". In questo senso è certamente corretto affermare che la concezione semantica della verità di Tarski «risulta essere la teoria della verità più adeguata anche in ambito giudiziario»: così G. Ubertis, Conoscenza fattuale, cit., p. 82 (ma v. già Id., Fatto e valore nel sistema probatorio penale, Giuffré, 1979, p. 91 s., nota 30).

16 L'argomento "ad ignorantiam" è appunto la fallacia argomentativa che consiste nel «conclude[re] che una proposizione è vera sulla base del fatto che non ci sono prove che sia falsa»: così A. Iacona, L'argomentazione, Einaudi, 2005, p. 111. 
tire $^{17}$. La conclusione che se ne trae è la stessa cui giungevano cent'anni fa Luigi Lucchini e Pasquale Tuozzi: nel processo penale, «l'assunto da dimostrarsi vero è quello soltanto che concerne la colpabilità» ${ }^{18}$; la sola verità da scoprire e reprimere è la «triste verità» della colpevolezza ${ }^{19}$.

Si può dunque liberare il campo da un primo equivoco. Non v’è dubbio che il processo penale soffra limiti cognitivi che spesso impediscono al giudice di pervenire a un'affermazione veritiera di responsabilità. La sua stessa struttura è intrinsecamente antiepistemica: ci sono tempi da rispettare, preclusioni, decadenze ${ }^{20}$. Molte regole di esclusione probatoria sono intese a salvaguardare valori che non sono affatto «connessi con la ricerca della verità» ${ }^{21}$ (basti pensare al divieto di utilizzare l'intercettazione telefonica non autorizzata o compiuta fuori termine, o alla facoltà, concessa ad alcuni testimoni, di opporre il segreto $^{22}$ ): e lo stesso criterio della colpevolezza beyond any reasonable

17 Cfr. G. Lozzi, Lezioni di procedura penale, Giappichelli, 2011, p. 599.

18 L. Lucchini, Elementi di procedura penale, Barbéra Editore, 1920, p. 14: «si domanda: la constatazione del vero dee trovar la sua espressione tanto nell'accertamento della colpabilità, per potersi condannare, quanto in quello dell'innocenza, per potersi prosciogliere? Qui deesi semplicemente ritornare con la mente alla ragion d'essere del procedimento, [che è] fondamentalmente istituito per ricercare e punire i delinquenti, non per discernere e accertare gli onesti. [.... L'assunto da dimostrarsi vero è quello soltanto che concerne la colpabilità. [...] Positivamente dee dimostrarsi la reità del giudicabile; basterà che tale dimostrazione non sia raggiunta, per doversi accogliere la tesi negativa contraria». Dunque, «quando si dice "scoperta della verità" nel processo penale si ha da intendere: dimostrazione della colpabilità dell'imputato rispetto a quel dato reato che gli si attribuisce».

19 P. Tuozzi, Principii del procedimento penale italiano, Stabilimento Ripografico M. D’Auria, 1909, p. 4.

20 In ambito processualcivilistico, il concetto è sottolineato con forza da B. Cavallone, In difesa della veriphobia (considerazioni amichevolmente polemiche su un libro recente di Michele Taruffo), in Riv. dir. proc., 2010, p. 22, che parla del processo come di una macchina cognitiva «inevitabilmente rigida, formalistica, ludica [...], inidonea a produrre gli stessi esiti di 'verità' che è legittimo aspettarsi da un laboratorio scientifico dotato di finanziamenti di entità e durata illimitate».

21 Cfr. M. Damaška, Il diritto delle prove alla deriva, Il Mulino, 2003, p. 24 s., che definisce "estrinseche" tali regole.

${ }^{22}$ Altre regole di esclusione probatoria sono invece senz'altro "intrinseche", ossia funzionali alla ricerca del vero. Lo è, ad esempio, il divieto di utilizzare le prove acquisite in difetto di contraddittorio: cfr. per tutti P. Ferrua, 
doubt impone uno sforzo giustificativo maggiore di quello normalmente richiesto per dichiarare vero un enunciato ${ }^{23}$. Tutto questo impedisce certamente al giudice penale di 'dire la verità' in molti casi nei quali lo storico o il giornalista potrebbero agevolmente permetterselo. Ma ciò non significa affatto che, quando il giudice perviene all'affermazione di responsabilità dell'imputato, la 'sua' verità sia una verità minore, formale, convenzionale, priva di valore logico-conoscitivo, non all'altezza di quella dello storico, del giornalista o dello scienziato ${ }^{24}$. Per sostenere questa tesi bisognerebbe in primo luogo dimostrare che l'incompletezza dell'accertamento non genera dubbi ragionevoli: ma esistono buoni motivi per ritenere che non sia così ${ }^{25}$. E bisognerebbe dimostrare che le regole estrinseche di esclusione probatoria valgono anche a danno dell'imputato, ossia che il giudice sarebbe obbligato a condannare anche in presenza di un'inconfutabile prova d'alibi, allorché, ad esempio, tale prova fosse stata ottenuta per mezzo di un'intercettazione non autorizzata o intempestiva. Ma neppure questa è un'impresa agevole: «l'accertamento dell'innocenza», sostiene Franco Cordero, «è una posta troppo importante per essere sacrificata agli idoli della procedura» ${ }^{26}$; e mentre

Contraddittorio e verità nel processo penale, in Studi sul processo penale, II, Anamorfosi del processo accusatorio, Giappichelli, 1992, p. 47 s.; Id., Anamorfosi del processo accusatorio, ivi, p. 184 s. Per un'opinione radicalmente difforme v. tuttavia M. Taruffo, La semplice verità, cit., p. 107 s., per cui «se vi è una cosa che al processo adversary non interessa in alcun modo, questa è proprio la verità», ed «è lecito dubitare che lo scontro delle parti, e il conflitto tra le rispettive versioni dei fatti, sia davvero un modo efficiente - o addirittura il più efficiente - per la scoperta della verità».

Sul punto A. Dershowitz, Dubbi ragionevoli. Il sistema della giustizia penale e il caso O. J. Simpson, Giuffrè, 2007, p. 28 s.

Per analoghi rilievi, nel contestare l'utilità e la fondatezza della distinzione tra verità "processuale" e verità "reale", M. Taruffo, La semplice verità, cit., p. 84 . In argomento rinviamo a F. Caprioli, L'accertamento della responsabilità penale "oltre ogni ragionevole dubbio", in Riv. it. dir. proc. pen., p. 59 s., 85 s.

F. Cordero, Prove illecite, in Tre studi sulle prove penali, Giuffrè, 1963, p. 171: il divieto di valutare la prova irritualmente acquisita troverebbe «una deroga nel caso in cui dalla risultanza che dovrebbe essere ammessa emergano illazioni favorevoli alla difesa»; più esattamente, il divieto probatorio si trasformerebbe in «una regola legale decisoria, che vieta al giudice d'assumere certe acquisizioni a premessa di una condanna». Nello stesso senso G. Lozzi, Lezioni, cit., p. 197, nonché, da ultimo, D. Vicoli, La "ragionevole durata" delle indagini, Giappichelli, 2012, p. 178 s. 
per assolvere, scriveva Francesco Carrara, è sufficiente la verità formale oppure quella sostanziale, «per la condanna è necessaria la verità formale e quella sostanziale al tempo stesso» ${ }^{27}$.

3. Ma veniamo al secondo assunto: la verità del processo sarebbe una verità minore perché all'accertamento del fatto di reato - come dimostra lo stesso canone decisorio del dubbio 'ragionevole' - non si può che pervenire in termini probabilistici.

Nel tentativo di valutarne la fondatezza, conviene prendere le mosse da un interrogativo tranchant. E' davvero indispensabile occuparsi della 'verità' delle decisioni del giudice? C'è qualche valida ragione per estendere i confini dell'indagine oltre la congruenza del discorso giustificativo che sorregge tali decisioni? In fondo, che si possa in qualche modo 'fare a meno' del concetto di verità è un'idea tutt'altro che peregrina nell'attuale dibattito filosofico: e chi la difende si serve di argomenti che potrebbero facilmente sedurre anche lo studioso del processo penale.

27 Questa l'opinione di Carrara, che rifletteva sul divieto imposto al giudice di decidere in facto avvalendosi della propria scienza privata: «il giudice [...] non può condannare colui che non è in processo provato colpevole, quantunque esso giudice sia certo, come privato, della di costui colpevolezza, [...] ma può assolvere per la sua certezza privata anche a dispetto di tutte le prove processuali che a lui constino erronee»; e «questa conclusione io la formulerei così: si deve assolvere secondo la verità sostanziale, ma per condannare è necessaria la verità sostanziale e formale al tempo stesso» (F. Carrara, Programma del corso di diritto criminale. Del giudizio criminale, con una selezione dagli Opuscoli di diritto criminale, Il Mulino, 2004, p. 172). Affermando che «si deve assolvere secondo la verità sostanziale», Carrara allude alla sola ipotesi in cui il giudice abbia la scienza privata dell'innocenza dell'imputato nonostante le prove acquisite in giudizio dimostrino la colpevolezza: ma nel caso inverso, sarebbe la verità formale a prevalere. In altre parole, per assolvere è sufficiente la verità sostanziale dell'innocenza in contrasto con quella formale (le prove acquisite nel processo dimostrano la colpevolezza ma il giudice sa che l'imputato è innocente) oppure la verità formale dell'innocenza in contrasto con quella sostanziale (il giudice sa che l'imputato è colpevole ma le prove acquisite nel processo dimostrano l'innocenza): per condannare, verità formale e verità sostanziale della colpevolezza devono coincidere (le prove acquisite nel processo devono dimostrare la colpevolezza $e$ il giudice non deve sapere che l'imputato è innocente). 
Ciò vale in primo luogo per le teorie logico-semantiche che tentano di 'sgonfiare' la questione della verità ${ }^{28}$ dimostrando che il termine 'vero' non svolge mai funzioni genuinamente predicative, ossia che, in definitiva, la verità non è una proprietà di alcunchée ${ }^{29}$. Il riferimento è all'opinione secondo cui l'affermazione 'è vero che Cesare fu assassinato' sarebbe semanticamente equivalente all'affermazione 'Cesare fu assassinato' (c.d. teoria della ridondanza) ${ }^{30}$ : opinione cui vengono spesso accostati sia l'orientamento (c.d. decitazionista) che riconduce il predicato di verità a un semplice dispositivo di devirgolettatura ${ }^{31}$, sia, talora, la stessa teoria dei bicondizionali di Tarski ${ }^{32}$. Come si è accennato, il processualista è chiamato ad affrontare il problema della verità da un angolo visuale che lo rende particolarmente vulnerabile a suggestioni di questo tipo. La struttura della decisione penale di condanna è infatti quella tipica del meccanismo di ascesa e discesa semantica: chiamato a pronunciarsi sull'imputazione - ossia ad occuparsi, anziché della realtà, di un enunciato che tenta di descriverla ${ }^{33}-$, il giudice che dichiara re-

28 To deflate: di qui l'etichetta di teorie deflazionistiche. Cfr. F. D’Agostini, Introduzione alla verità, cit., p. 76 s.

29 Sul punto G. Volpe, Teorie della verità, cit., p. 256 s.

30 L'espressione "è vero" verrebbe dunque usata soltanto "per enfasi o per ragioni stilistiche, o per indicare la posizione che l'affermazione occupa nella nostra argomentazione»: F.P. Ramsey, Fatti e proposizioni, in I fondamenti della matematica e altri scritti di logica, Feltrinelli, 1964, p. 159 s.

31 L'operazione sarebbe funzionale alla 'discesa semantica' dal linguaggio in cui parliamo degli enunciati al linguaggio in cui parliamo del mondo: sul punto G. Volpe, Teorie della verità, cit., p. 263 s.

32 Sui rapporti tra la teoria di Tarski e le teorie ridondandistiche e decitazionistiche il dibattito, in realtà, è aperto. Lo stesso W.V.O. Quine, Parola ed oggetto, Il Saggiatore, 1996, p. 36, rinvia in nota a Tarski per lo «sviluppo classico» del tema secondo cui «dire che l'enunciato "Bruto ha ucciso Cesare" è vero [...] equivale, di fatto, semplicemente a dire che Bruto ha ucciso Cesare». Per una critica alla diffusa opinione che «il lavoro di Tarski sulla verità non sia altro che [...] un rafforzamento dell'intuizione di Ramsey, [ossia] un miglioramento tecnico di una teoria fondamentalmente ridondandista», cfr. tuttavia D. Davidson, Sulla verità, Laterza, 2006, p. 13 s.; in prospettiva analoga M. Dummett, Verità e passato, Raffaello Cortina Editore, 2006, p.125, che respinge vigorosamente le critiche di "disinvolto decitazionismo" rivolte a Tarski da R. Rorty, La verità è una meta della ricerca?Donald Davidson contro Crispin Wright, in Verità e progresso. Scritti filosofici, Feltrinelli, 2003, p. 22. 
sponsabile l'imputato sale al livello semantico in cui si parla dell'enunciato contenuto nell'atto d'accusa per poi immediatamente ridiscendere al linguaggio in cui si parla del reale ${ }^{34}$. L'idea che il predicato 'vero' costituisca un semplice dispositivo di devirgolettatura ('l'imputato è colpevole' è vero perché l'imputato è colpevole) sembra dunque particolarmente congeniale a una definizione processuale della verità, così come - lo si è già detto - l'idea che tale predicato faccia da tramite tra due linguaggi (il linguaggio dell'imputazione e quello della decisione) anziché tra il linguaggio e il mondo. Interrogarsi sulla verità delle affermazioni di responsabilità penale significa, inoltre, concentrare l'analisi su enunciati che contengono attribuzioni di verità a proposizioni date esplicitamente ('è vero che Tizio ha commesso il reato X') e non soltanto evocate per implicito (come avviene in enunciati del tipo 'tutto quello che hai sostenuto è vero', nei quali, come si usa dire, l'attribuzione di verità non è rivelatrice $)^{35}$. Ciò consente allo studioso della verità dei giudizi di condanna di evitare il principale ostacolo che si frappone all'accoglimento delle teorie deflazionistiche, rappresentato dalla difficoltà di sopprimere impunemente il termine 'vero' dagli enunciati in cui, appunto, «non ci sono virgolette da rimuovere ${ }^{36}$ perché l'attribuzione di verità è cieca ${ }^{37}$.

Fin qui le teorie semantiche. Ma il ripudio del concetto di verità viene talora argomentato su basi esclusivamente pragmatiche: la distinzione tra verità e giustificazione - ha scritto, ad esempio, Richard Rorty - «è una differenza che non fa nessuna differenza quando si tratta di decidere che cosa fare. Se ho dei dubbi concreti e specifici sulla verità di una delle mie credenze li posso risolvere solo chiedendomi se tale credenza ha una giustificazione adeguata, trovando e valutando nuovi pro e contro. Non posso mettere da parte la giustificazione e limitare la mia attenzione alla sola verità: quando il problema è cosa devo credere

34 Dicendo che è vero quanto affermato dal pubblico ministero, ossia dicendo «Tizio ha commesso il reato X' è vero», il giudice afferma che Tizio ha commesso il reato X: tolte le virgolette, l'attenzione ritorna dall'enunciato al mondo.

35 Il termine, mutuato da G. Volpe, Teorie della verità, cit., p. 261, si deve a W. Künne, Conception of Truth, Clarendon Press, Oxford, 2003, p. 52. D. Davidson, Sulla verità, cit., p. 15.

37 In argomento cfr. F. D'Agostini, Introduzione alla verità, cit., p. 77 s., e, per una dettagliata analisi, G. Volpe, Teorie della verità, cit., p. 255 s. 
adesso, valutare se una credenza è vera e valutare se è giustificata sono la stessa $\operatorname{cosa} »^{38}$. Anche questa è un'idea che potrebbe facilmente attecchire sul terreno del processo penale e delle sue dinamiche cognitive. Non v'è dubbio che l'efficienza di un sistema processuale si misuri sul grado di giustificazione razionale delle decisioni adottate assai più che sul loro livello di (pretesa) corrispondenza al vero. Anche nei riti ordalici la "giustizia" del verdetto era connessa a una ritenuta verità delle accuse rivolte all'imputato ${ }^{39}$ : e l'ossessione della verità è notoriamente un tratto caratteristico di modelli processuali penali - quelli di matrice inquisitoria - che si sono rivelati deludenti sul piano cognitivo e funzionale prima ancora che ideologicamente poco raccomandabili.

Fortemente suggestiva per il processualista potrebbe infine rivelarsi l'opinione di chi - pur essendo convinto che della nozione di verità non si possa fare a meno ${ }^{40}$ - ritiene che per definire vero un enunciato non ci si dovrebbe interrogare sulla sua corrispondenza alla realtà, ma sulla possibilità di trovarne una giustificazione ${ }^{41}$ : o di chi ritiene, in analoga prospettiva, che potrebbero dirsi vere soltanto le proposizioni che risulterebbero giustificate (e sarebbe, pertanto, razionale accettare) in condizioni epistemiche ideali $^{42}$. Anche simili proposte ricostruttive - riconducibili al

38 R. Rorty, La verità è una meta della ricerca?, cit., p. 21 (corsivi aggiunti).

39 Cfr. M. Taruffo, La semplice verità, cit., p. 80.

40 Secondo M. Dummett, Verità e passato, cit., p. 29, 38, 45, «i concetti di verità e significato sono inestricabilmente connessi [e] devono essere spiegati insieme, [senza che nessuno possa essere] considerato prioritario rispetto all'altro». In questa prospettiva il concetto di verità resterebbe indispensabile, perché in sua assenza non si saprebbe come definire ciò «a cui si impegna» l'autore di un'asserzione e ciò «di cui è garantita la trasmissione dalle premesse alla conclusione di un argomento deduttivamente valido».

41 Così M. Dummett, Verità e passato, cit., passim, spec. p. 29, 38, 41, 130, 131: dobbiamo «considerare vero un asserto se è o era possibile trovarvi una giustificazione. [...] Quello che non possiamo fare è considerare la verità come una proprietà che ogni asserto determinatamente possiede o di cui è determinatamente privo, indipendentemente dagli strumenti che potremmo mai avere in nostro possesso per decidere» in un senso o nell'altro. Come avverte F. D’Agostini, Introduzione alla verità, cit., p. 90, l'epistemicismo di Dummett «è anzitutto una teoria del significato, o meglio della comprensione del significato»: «capisco p quando so come stabilire che l'enunciato p è vero».

42 Così H. Putnam, Ragione, verità e storia, Il Saggiatore, 1981, p. 63, che ha in seguito notoriamente mutato opinione (cfr. Id., Realismo e senso comune, in 
genus delle teorie epistemiche della verità - potrebbero felicemente adattarsi alla verità del processo. Riferendosi a eventi del passato, insuscettibili di osservazione diretta, le proposizioni della cui verità si discute in ambito processuale sono necessariamente inferite da altre proposizioni ${ }^{43}$ : poiché il loro essere vere non risulta dal confronto con esperienze 'pure', immuni da contaminazioni concettuali, la prospettiva sembrerebbe favorevole all'abbandono di pretese corrispondentiste ${ }^{44}$. Le concezioni epistemiche si direbbero inoltre connaturali a un sistema cognitivo che del metodo di accertamento della verità «fornisce [un] dettagliato paradigma, vincolando gli operatori al suo rispetto, quali che siano i riflessi sulla ricostruzione dei fatti» ${ }^{45}$; altrimenti detto, «proprio nel processo, più che altrove, si sarebbe tentati di risolvere la verità nel metodo $»^{46}$.

Un oculato governo delle proprie risorse intellettuali sembrerebbe dunque suggerire allo studioso del processo penale di accantonare la nozione di verità per dedicarsi in via prioritaria, se non esclusiva, all'analisi del concetto di giustificazione: ossia per chiedersi, più modestamente, a quali condizioni una sentenza di condanna può dirsi derivata in modo convincente da premesse plausibilii ${ }^{47}$. E' su questo terreno,

Aa.Vv., Bentornata realtà, cit., p. 8 s.). Analoga la posizione di chi giudica vere le proposizioni che verrebbero credute da tutti i membri della comunità scientifica qualora l'indagine su di esse non fosse suscettibile di ulteriori approfondimenti: è la teoria del 'limite ideale' esposta da C.S. Peirce, Definitions of Truth, in Collected Papers, a cura di C. Hartshorne, P. Weiss e A. Burks, Belknap Press, Cambridge, vol. V, p. 394. Giudica «vuota e inafferrabile» la verità concepita in questi termini P. Ferrua, Contraddittorio e verità, cit., p. $68 \mathrm{~s}$.

43 Cfr. M. Taruffo, La prova dei fatti giuridici. Nozioni generali, Giuffré, 1992, p. 234 s.; G. Ubertis, Conoscenza fattuale, cit., p. 83.

44 Come rileva G. Volpe, Teorie della verità, cit., p. 126, 142, è stata proprio la constatazione che «una proposizione può essere inferita soltanto da altre proposizioni» (ossia il rilievo che «entità prive di componenti concettuali quali sarebbero, se esistessero, le esperienze 'pure' potrebbero esercitare un'azione causale sulle nostre credenze, ma non potrebbero in alcun modo giustificarne il contenuto») a «indu[rre] alcuni filosofi a preferire alla teoria della corrispondenza la teoria coerentista o la teoria pragmatica della verità». P. Ferrua, Contraddittorio e verità, cit., p. 66. Così ancora P. Ferrua, Contraddittorio e verità, cit., p. 66.

$\mathrm{E}$ ' in questo senso che si parlerà nel testo di asserzioni 'giustificate': non, dunque, riferendosi alle asserzioni giustificate in quanto (comunque) argomentate, o riferendosi alle asserzioni giustificate in quanto vere. Su queste tre diverse accezioni del termine cfr. D. Marconi, Per la verità, cit., p. $11 \mathrm{~s}$. 
del resto, che la riflessione filosofica sembra attualmente consegnare al processualista i più delicati interrogativi. Il riferimento è al problema del c.d. 'relativismo epistemico', cioè all'idea che nessun sistema epistemico sarebbe in grado di spiegare se non dal proprio interno quando una certa credenza o una certa asserzione può dirsi giustificata ${ }^{48}$ : ma anche alle difficoltà che il nostro sistema epistemico - fondato sui principi dell'osservazione, della deduzione, dell'induzione, dell'inferenza alla spiegazione migliore ${ }^{49}$ - continua a incontrare nel giustificare se stesso, ossia al fatto che secondo i filosofi della scienza noi ancora «non dispo[rremmo] di una definizione del tutto soddisfacente del 'concetto qualitativo' di conferma, vale a dire di una soddisfacente caratterizzazione dei casi nei quali una data evidenza e conferma (costituisce una 'buona evidenza' per) un'ipotesi $h »^{50}$. E' qui che rischiano di scricchiolare le fondamenta del sistema cognitivo penale, a partire dall'idea stessa - implicita nell'art. 606 lett. e c.p.p. - che sia possibile vagliare la 'logicità' e la 'non contraddittorietà' dell'apparato giustificativo delle sentenze.

Perché, dunque, interessarsi alla verità dei verdetti giudiziali? Perché la più attenta dottrina processualistica continua a farlo, difendendo strenuamente l'idea della verità processuale come corrispondenza ai fatti ${ }^{51}$ ?

48 E' il concetto che Thomas Nagel illustra esemplarmente dicendo che se ci fosse qualcuno che decide questioni fattuali o pratiche consultando le foglie di tè, e noi gli chiedessimo di dimostrare la razionalità del suo metodo, lui risponderebbe consultando ulteriormente le foglie di tè: il che è quanto faremmo, in fondo, anche noi (T. Nagel, L'ultima parola: contro il relativismo, Feltrinelli, 1999, p. 29). Sul punto, per una convinta difesa dell'oggettivismo circa la giustificazione, P.A. Boghossian, Paura di conoscere, cit., p. 39 s., 77 s., nonché D. Marconi, Per la verità, cit., p. 50 s.

P.A. Boghossian, Paura di conoscere, cit., p. 84 s.

R. Festa, Induzione, probabilità e verisimilitudine, in G. Giorello, Introduzione alla filosofia della scienza, Bompiani, 1999, p. 296.

51 Nel senso che le proposizioni espresse dalle decisioni giudiziali potrebbero definirsi 'vere' solo quando esistano i fatti ai quali esse corrispondono cfr. ad esempio P. Comanducci, La motivazione in fatto, in AA.VV., La conoscenza del fatto nel processo penale, Giuffré, 1992, p. 238 s.; L. Ferrajoli, Diritto e ragione, cit., p. 18 s.; M. Taruffo, La prova dei fatti giuridici, cit., p. 145; Id., Fatto, prova e verità, p. 316 s. Occorre notare che tutti questi autori si richiamano alla teoria semantica della verità di Tarski, muovendo concordemente dalla premessa - tutt'altro che scontata (cfr. D. Marconi, Realismo minimale, in 
4. In termini consapevolmente paradossali ${ }^{52}$, qualcuno ritiene che al ripudio di ogni tentazione deflazionistica o giustificazionistica si dovrebbe pervenire, in ambito processuale penale, per ragioni di carattere pragmatico. In primo luogo, perché confondere la verità di un giudizio penale con la sua giustificazione (e, segnatamente, con la sua coeren$\mathrm{za}^{53}$ e la sua accettabilità giustificata) rischierebbe di aprire la strada a decisioni penali fondate su «criteri di moralità, o di convenienza o di efficienza», mentre «nel diritto penale, la sola giustificazione accettabile delle decisioni è quella rappresentata dalla verità dei loro presupposti giuridici e fattuali, intesa 'verità' proprio nel senso di 'corrispondenza' il più possibile approssimativa della motivazione alle norme applicate e ai fatti giudicati» ${ }^{54}$. In secondo luogo, perché difendendo l'idea che un enunciato può essere falso «pur ottemperando a tutti i requisiti stabiliti per la sua asseribilità ${ }^{55}$ si tutelerebbe il diritto del condannato di proclamarsi innocente anche quando la tesi della sua innocenza sia stata «respinta in tutti i gradi di giudizio e [sia] in contrasto con tutte le prove disponibili» ${ }^{56}$. Su entrambi i versanti, non si tratterebbe, beninteso, di coltivare illusioni sulla possibilità offerta al giudice di attingere a qualche forma di verità 'oggettiva' o 'assoluta': la verità processuale, basata sull'induzione, non potrebbe che rimanere una verità probabile, approssimativa, allo stato delle conoscenze ${ }^{57}$. Il richiamo alla verità come cor-

Aa.Vv., Bentornata realtà, cit., p. 120) - che essa postuli una corrispondenza tra il linguaggio e il reale. Nega, condivisibilmente, tale premessa, giudicando tuttavia «inevitabili sul terreno del processo penale [...] le implicazioni ontologiche di aggancio tra linguaggio e mondo», e «in qualche modo implicite [...] le esigenze corrispondentiste [...] negli enunciati fattuali formulati nel processo», P. Ferrua, Contraddittorio e verità, cit., p. $71 \mathrm{~s}$.

Ammette il paradosso P. Ferrua, Contraddittorio e verità, cit., p. 74.

Sulle teorie coerentiste della verità cfr. F. D'Agostini, Introduzione alla verità, cit., p. $55 \mathrm{~s}$.

L. Ferrajoli, Diritto e ragione, cit., p. $42 \mathrm{~s}$.

P. Ferrua, Contraddittorio e verità, cit., p. 67.

L. Ferrajoli, Diritto e ragione, cit., p. 41.

L. Ferrajoli, Diritto e ragione, cit., p. 23, 26 s., 41: «quando si afferma la 'verità' di una o più proposizioni, la sola cosa che si dice è che queste sono (plausibilmente) vere per quanto ne sappiamo, ossia rispetto all'insieme delle conoscenze confermate che possediamo»; «come in tutte le inferenze induttive, anche nell'inferenza storiografica e giudiziaria la conclusione ha solo il valore di un'ipotesi probabilistica in ordine alla connessione causale tra il fatto as- 
rispondenza sarebbe tuttavia indispensabile per «fissare un'idea guida, un principio regolativo» in grado di soddisfare le due riferite esigenze (scongiurare ogni possibile «asservimento [dei criteri decisionali adottati dal giudice] a finalità inaccettabili per la giurisdizione, come la ricerca della soluzione 'utile'» ${ }^{58}$, e ripudiare ogni dogmatismo epistemologico).

Sono rilievi solo in parte condivisibili: e non solo per quanto si dirà nel prossimo paragrafo sui rapporti tra verità e certezza e sull'esigenza di non 'drammatizzare' la questione della verità ${ }^{59}$. Due le possibili obiezioni. In primo luogo, per contestare la verità di un assunto razionalmente giustificato non sembra indispensabile fare professione di corrispondentismo. Il concetto di verità può essere usato in questa accezione 'cautelativa' senza alcuna implicazione realista ${ }^{60}$ : e anche l'opinione di chi fa coincidere verità e giustificazione solo al realizzarsi di condizioni epistemiche ideali ${ }^{61}$ si direbbe immune da ogni dogmatismo epistemolo-

sunto come provato e l'insieme dei fatti addotti come probatori». Dunque la verità sarebbe «caratterizzabile, sul piano semantico, come corrispondenza solo per quanto ne sappiamo e solo in misura approssimativa». Per considerazioni analoghe v. P. Comanducci, La motivazione in fatto, cit., p. 238 s. (in campi come la storia e il diritto, «questo senso di 'vero', cui si richiama la teoria semantica della verità come corrispondenza, può solo servire come limite ideale e come pietra di paragone [...] Qui ‘vero' può voler dire soltanto ‘più probabile allo stato delle conoscenze attuali'»), e P. Ferrua, Contraddittorio e verità, cit., p. 53 s., 74 nota 80 (essendo la decisione del giudice basata su ragionamenti di tipo induttivo, «le prove non possono mai [...] garantire esiti di assoluta certezza, di verità oggettiva»; l'induzione «non esprime mai una verità necessaria, ma soltanto un certo grado di probabilità»; dunque «la 'corrispondenza' può essere predicata solo in termini di probabilità»).

P. Ferrua, Contraddittorio e verità, cit., p. 67, 69. Sul valore regolativo della verità come corrispondenza ai fatti v. anche M. Taruffo, Fatto, prova e verità, cit., p. 317.

L'espressione è di D. Marconi, Per la verità, cit., p. 34 s.

Secondo Richard Rorty, quando diciamo ‘è giustificato, ma forse non è vero', non stiamo «contrapponendo le ragioni di credere o di giustificare un enunciato alla maniera in cui le cose sono 'in realtà'», non introduciamo «una distinzione tra qualcosa di umano e qualcosa di non umano, ma tra situazioni diverse nelle quali possono trovarsi gli esseri umani: la situazione presente, nella quale la credenza pare giustificata, e una situazione ipotetica, in futuro, in cui si considererà che questo non sembri più essere il caso» (così, da ultimo, P. Engel - R. Rorty, A cosa serve la verità, Il Mulino, 2007, p. 60). Sul punto v. D. Marconi, Per la verità, cit., p. 17. 
gico se trasferita in ambito processuale (non potendo mai definirsi ideali le condizioni epistemiche che si realizzano in tale ambito). In secondo luogo, non v'è dubbio che debba essere bandita dal processo penale qualunque decisione fondata su criteri volontaristici o di convenienza anziché sull'accertamento del vero: e non v'è dubbio che il legislatore del 1988 abbia commesso un errore, da questo punto di vista, nel ripudiare normativamente il concetto di verità ${ }^{62}$ (così come, in generale, è un grave equivoco contrapporre i processi di stampo accusatorio ai processi di stampo inquisitorio sul presupposto che gli uni punterebbero al rispetto delle garanzie individuali anche a scapito della verità, gli altri all'accertamento della verità anche a scapito delle garanzie individuali $\left.{ }^{63}\right)$. Rimane tuttavia da dimostrare che la verità intesa come corrispondenza ai fatti sia la teoria più idonea ad accreditare simili posizioni ${ }^{64}$. Si potrebbe in-

62 V. supra, § 1. Severissimo, al riguardo, il giudizio critico di M. Nobili, L'immoralità necessaria. Citazioni e percorsi nei mondi della giustizia, Il Mulino, 2009, p. 212: «la repulsione per l'art. 299 c.p.p. del 1930 (la ricerca della verità) cadeva su tutt'altro problema (il dibattito, ora confuso e frainteso, sulla funzione della sola fase istruttoria). Eppure [qualcuno] desunse da lì che - con il nuovo processo - non saremmo più passati attraverso l'accertamento di verità! [...] Sono palesi le implicazioni. Cacciando via quel parametro, ci si sposta [...] nei mondi della volontà, a loro volta contigui o coincidenti con quelli del potere. Ma fa tremare: Tizio commise il fatto criminoso, perché così è "preferibile"; perché così si vuole. [...] Tremende sono le conseguenze di una concezione volontaristica del procedere e del punire». Dunque «la "verità" [...] resta lo strumento immancabile per gli scopi del giudizio penale» (ivi, p. 208).

63 Sul punto, limpidamente, P. Ferrua, Contraddittorio e verità, cit., p. 47 s.; Id., Anamorfosi del processo accusatorio, ivi, p. 184 s., nonché M. Nobili, L'immoralità necessaria, cit., p. 216 («idea con ben poco o nessun fondamento»).

64 Secondo P. Ferrua, Contraddittorio e verità, cit., p. 74 s., non potrebbe che abbracciare l'ideale corrispondentista chiunque intenda salvaguardare i fondamenti stessi della responsabilità penale: come si potrebbe infatti giustificare la pretesa punitiva, se si ritenesse «che l'enunciato di colpevolezza, sulla cui base è inflitto il carcere, non ha con la realtà, con ciò che 'è stato', alcun rapporto di adeguatezza o di corrispondenza»? Ma pur gravide di implicazioni antirealistiche (cfr. G. Volpe, Teorie della verità, cit., p. 185 s., e F. D’Agostini, Introduzione alla verità, cit., p. 89 s.), le teorie epistemiche della verità hanno se non altro il merito di sottrarsi ai paradossi scettici che derivano dall'attribuzione di un valore di verità a proposizioni come 'il mondo e tutto ciò che contiene, memorie e fossili inclusi, è venuto all'esistenza cinque minuti fa'. L'eventualità che esistano verità siffatte - anch'esse apertamente contrastanti con la logica del sistema punitivo - è infatti addirittura inintelligibile per chi identifica verità e 
fatti sostenere il contrario: a favorire l'ingresso di elementi pragmatici e utilitaristici nella formazione dei convincimenti giudiziali (come, in genere, delle credenze del ricercatore) rischia di essere proprio l'idea dell'irriducibilità epistemologica della verità che informa i paradigmi realisti e corrispondentisti ${ }^{65}$, ossia l'idea che esista un'incolmabile lacuna tra ciò che è vero e ciò che saremmo in grado di riconoscere come tale anche in condizioni epistemiche ideali. Non è un caso che la tesi quiniana della necessaria 'sottodeterminazione' di qualsiasi credenza rispetto alle prove disponibili venga talora giudicata responsabile di portare acqua al mulino del c.d. 'costruttivismo circa la spiegazione razionale'66, ossia di accreditare l'opinione che non sarebbe «mai possibile spiegare perché crediamo quello che crediamo solamente sulla base del fatto che abbiamo evidenze a favore», in quanto «anche i nostri bisogni e interessi contingenti [andrebbero necessariamente] presi in considerazione ${ }^{67}$. Nel tentativo di respingere questa accusa, i difensori del corrispondentismo sottolineano la natura puramente logica della tesi di Quine: sostenere che «l'evidenza è formalmente compatibile con più di una teoria» - si fa rilevare - «non equivale a dire che è razionalmente compatibile con più di una teoria» ${ }^{68}$. Ma così l'attenzione torna inesorabilmente a spostarsi dalla verità delle proposizioni alla loro giustificazione. Con rilievi analoghi si replica all'obiezione secondo cui la teoria della verità come corrispondenza, per i suoi esiti radicalmente scettici, non potrebbe costituire un'adeguata guida per la ricerca ${ }^{69}$ : l'obiezione sarebbe valida, si osserva, solo se la falsità della proposizione accreditata dalla migliore

giustificazione: l'esistenza di mondi scettici come quello descritto viene ritenuta «metafisicamente impossibile», benché «logicamente possibile», proprio perché, se esistessero, «le proposizioni che descrivono [simili mondi] non potrebbero essere riconosciute come vere» (cfr. G. Volpe, ivi, p. 189).

Definisce 'realismo minimale' tale posizione, citando Michael Williams, D. Marconi, Realismo minimale, cit., p. 117, nota 2.

66 Insieme con la teoria delle ipotesi ausiliarie di Pierre Duhem, cui viene spesso accostata: cfr. P.A. Boghossian, Paura di conoscere, cit., p. 147 s.

67 P.A. Boghossian, Paura di conoscere, cit., p. 133.

68 P.A. Boghossian, Paura di conoscere, cit., p. 149. V. anche G. Volpe, Teorie della verità, cit., p. 131 s., e D. Marconi, Per la verità, cit., p. 32 s.

69 Le teorie epistemiche del 'limite ideale' e della 'idealizzazione dell'accettabilità razionale' sarebbero invece perfettamente attrezzate allo scopo: cfr., per i necessari riferimenti, G. Volpe, Teorie della verità, cit., p. 162, 177 s. 
evidenza possibile fosse un'ipotesi che i corrispondentisti ritengono non soltanto logicamente sostenibile, ma anche fornita del medesimo grado di probabilità dell'ipotesi contraria ${ }^{70}$. Di nuovo torna in primo piano il concetto di accettabilità razionale delle credenze e si rende necessario tentare di delimitarne i confini. L'impressione, in definitiva, è che siano queste (la giustificatezza, la razionalità) le categorie concettuali su cui si deve lavorare per scongiurare le decisioni 'utili', le condanne 'preferibili', le derive volontaristiche. $E$ il lavoro non manca, perché si tratta innanzitutto di respingere le due offensive cui si è fatto cenno in precedenza ${ }^{71}$ : l'una condotta in nome del relativismo epistemico (si intenda o meno anche la verità nella sua accezione epistemica $)^{72}$, l'altra rivolta ai fondamenti del nostro sistema conoscitivo.

Ritorna dunque l'interrogativo di partenza: perché occuparci della verità delle decisioni penali, oltre che della loro giustificazione razionale?

5. Il più recente dibattito filosofico sui rapporti tra verità e giustificazione offre a questa domanda una semplice e convincente risposta. Ci dobbiamo occupare della verità - della verità intesa in senso corrispondentista e realista ${ }^{73}$, pur con tutte le difficoltà che questa tesi incontra $^{74}$ - perché la verità è uno degli ingredienti del concetto stesso di giustificazione razionale. Come scrive Bernard Williams, «un'affermazione giustificata è un'affermazione sostenuta da considerazioni che

70 Cfr. G. Volpe, Teorie della verità, cit., p. 163.

71 Sul punto rinviamo a F. Caprioli, La scienza "cattiva maestra": le insidie della prova scientifica nel processo penale, in Cass. pen., 2008, p. $3531 \mathrm{s.}$

72 Come puntualizza D. Marconi, Per la verità, cit., p. 54, 85 nota 8, benché gran parte dei sostenitori delle teorie epistemiche della verità siano anche relativisti sul piano epistemico (e finiscano, dunque, per essere relativisti anche in ordine alla verità), non occorre affatto essere corrispondentisti per ripudiare il pluralismo e il relativismo epistemico, ossia per ritenere che «ci siano criteri di giustificazione unici, o superiori a tutti gli altri».

73 Sul 'realismo aletico' come versione evoluta della teoria della corrispondenza, e sui suoi rapporti con il realismo metafisico, cfr. F. D’Agostini, Introduzione alla verità, cit., p. $86 \mathrm{~s}$.

${ }^{74}$ Per una puntuale rassegna delle obiezioni mosse al corrispondentismo - di natura analitica, epistemologica e semantica - cfr. G. Volpe, Teorie della verità, cit., p. 118-132, e F. D’Agostini, Introduzione alla verità, cit., p. 51 s. 
la favoriscono non semplicemente nel senso di renderla più attraente, ma nel senso specifico di dar ragione per ritenere che sia vera» ${ }^{75}$. E' questo rapporto di implicazione che indebolisce le teorie epistemiche e le teorie deflazionistiche della verità: come si può dire 'vero in quanto giustificato' (sia pure in condizioni epistemiche ideali), se ciò che è giustificato lo è in quanto si ha ragione di ritenere che sia vero ${ }^{76}$ ? E come si può dire 'non ci interessa la verità, solo la giustificazione', se c'è motivo di ritenere che «qualsiasi concetto di giustificazione sia direttamente o indirettamente tributario del concetto di verità» ${ }^{77}$ ?

Anche le decisioni del giudice penale vanno inquadrate in questo schema esplicativo. Dire 'ritengo provato oltre ogni ragionevole dubbio che Tizio abbia ucciso Caio' significa dire 'ho buone ragioni per ritenere vero che Tizio abbia ucciso Caio', perché accertare $X$ - questo è il punto - implica accertare che $X$ è vero. E' per questo che ha torto la Corte costituzionale quando lascia intendere che l'accertamento dei fatti' sarebbe, per il giudice penale, un compito meno gravoso o meno ambizioso della 'ricerca della verità'78.

S'intende che non esiste alcuna certezza (intesa in senso oggetti$\mathrm{vo}^{79}$ ) che il giudice, quando condanna, stia dicendo il vero, perché il ragionamento probatorio si nutre di inferenze che restano inevitabilmente probabilistiche. Ma questo non significa che la verità del processo penale sia una verità formale, convenzionale, relativa, né che sia un'entità inaccessibile, un inafferrabile miraggio. Come è stato esattamente osservato, non bisogna drammatizzare il tema della verità ${ }^{80} \mathrm{e}$, soprattutto, non biso-

75 B. Williams, Truth and Truthfulness, Princeton University Press, 2002, p. 129.

76 Nel senso che non è facile spiegare che cosa renda "ideale" una piattaforma epistemica «senza chiamare in causa quella stessa proprietà [la verità] di cui tale caratterizzazione dovrebbe aiutare a chiarire la natura», cfr. G. Volpe, Teorie della verità, cit., p. 185, cui si rinvia per ulteriori riferimenti.

77 D. Marconi, Per la verità, cit., p. 21.

78 V. supra, § 1 . Lo rileva anche D. Marconi, Per la verità, cit., p. 7 s., 151, che fa giustamente notare, al contrario, come accertare i fatti sia un'impresa molto più ardua che ricercare semplicemente la verità.

79 Per una distinzione tra verità e certezza fondata su una nozione di certezza marcatamente soggettiva, cfr. invece M. Taruffo, La semplice verità, cit., p. 85 s.

80 D. Marconi, Per la verità, cit., p. 34 s. Sull'«uso pesante o imbarazzato delle parole 'fatti', 'realtà' (e conseguentemente 'verità')» come «portato di una 
gna confondere verità e certezza. La verità - intesa come corrispondenza ai fatti - è alla portata di tutti: persone comuni e magistrati la dicono molto più spesso di quanto non si creda, pur non potendone essere certi. Né bisogna confondere certezza e conoscenza, cioè rassegnarsi allo scetticismo. La formula contenuta nell'art. 533 c.p.p. scolpisce perfettamente la distinzione: un conto è il dubbio 'ragionevole', un conto è il dubbio puramente scettico, quello che, a differenza del primo, «non ha motivazioni specifiche, legate a ciò che abbiamo motivo di pensare in una specifica situazione» ${ }^{81}$. Il dubbio scettico cancella ogni certezza: ma solo il dubbio ragionevole impedisce al giudice penale di coltivare credenze razionalmente giustificate e di attingere alla 'conoscenza' dei fatti.

Quando mancano dubbi ragionevoli circa la colpevolezza dell'imputato, noi dunque non possiamo che ritenere che il giudice stia dicendo la verità: non abbiamo ragioni per sostenere il contrario, perché «la possibilità di una ragione non è una ragione» ${ }^{82}$. Ciò naturalmente non significa indulgere ad alcuna forma di relativismo epistemico e, meno che mai, di relativismo circa la verità: non significa negare che esistano 'metacriteri' per stabilire se un asserto è o non è razionalmente giustificato, e non significa ritenere che un asserto vero per $\mathrm{X}$ potrebbe non essere vero per $\mathrm{Y}^{83}$. Per riprendere un felice

metafisica implicita, assunta dogmaticamente, e tributaria di un'epoca lontana», v. F. D’Agostini, Introduzione alla verità, cit., p. 234 s., 256.

D. Marconi, Per la verità, cit., p. 25.

D. Marconi, Per la verità, cit., p. 33, 36: «se una credenza è giustificata, abbiamo ragione di ritenerla vera (che è giustificata significa appunto questo): questa ragione non viene meno - ovviamente - per il fatto che la credenza può essere falsa. Una ragione per credere che $\mathrm{P}$ può essere scalzata solo da ragioni in senso opposto, non dalla mera possibilità che tali ragioni esistano [...] Fintantoché queste ragioni in senso contrario non vengono prodotte e non risultano prevalenti sulle nostre attuali giustificazioni, non solo siamo autorizzati a trattare le nostre credenze giustificate [nel senso di derivate in forma convincente da premesse plausibili] come conoscenze: sarebbe, alla lettera, irrazionale da parte nostra non farlo».

83 Sul relativismo in ordine alla verità cfr. ampiamente D. Marconi, Per la verità, cit., p. 50 s., che osserva come tale opinione possa essere sostenuta solo prestando congiuntamente adesione alla concezione epistemica della verità e al relativismo epistemico (ivi, p. 53 s.), oppure muovendo da premesse di relativismo concettuale, ossia negando che esista un modo in cui stanno le cose indipendente dagli schemi concettuali in cui noi le inquadriamo (ivi, p. 57 s.). 
esempio $^{84}$, decidere una controversia giuridica sul presupposto che fosse il sole a girare intorno alla terra era razionalmente giustificato, in età precopernicana, esattamente come lo è oggi decidere in senso contrario, perché, prima di Copernico, il dubbio che fosse la terra a girare intorno al sole non era un dubbio ragionevole ma un dubbio puramente scettico ${ }^{85}$. Ma poiché esistono ottimi argomenti contro il relativismo epistemico ${ }^{86}$, oggi noi sappiamo, e possiamo dimostrare, che le nostre ragioni sono migliori di quelle messe in campo dal giudice precopernicano, e possiamo dunque affermare che quella decisione era inveritiera ${ }^{87}$ - basata su premesse che erano false allora come lo sono oggi ${ }^{88}$.

Anche nella letteratura processualistica si parla di verità 'relativa' senza pervenire ad esiti di autentico relativismo: cfr. ad esempio M. Taruffo, La semplice verità, cit., p. 82; Id., Contro la veriphobia, cit., p. 1002 (la verità sarebbe relativa «nel senso che la conoscenza della verità è relativa al contesto in cui essa viene realizzata, al metodo con cui si svolge la ricerca e alla quantità e qualità di informazioni di cui si dispone e sulle quali tale conoscenza si fonda»: il che, come è ovvio, «non significa affatto svalutare la funzione della verità, e neppure significa adottare una concezione soggettivistica o relativistica di essa»).

Lo stesso non può dirsi per il cardinale Bellarmino, che poteva guardare dentro il cannocchiale di Galileo e non lo ha fatto: v. M. Taruffo, Contro la veriphobia, cit., p. 1002.

Cfr. P.A. Boghossian, Paura di conoscere, cit., p. 39 s., 77 s.

Ammette invece che la decisione del giudice precopernicano potrebbe essere ritenuta «inveritiera» solo «dal punto di vista dei posteri», B. Cavallone, In difesa della veriphobia, cit., p. 10, incorrendo nelle (giuste) critiche di «relativismo radicale riferito al problema della verità» di M. Taruffo, Contro la veriphobia, cit., p. 1002.

Un problema diverso è se quella sentenza sia oggi da ritenersi anche ingiustamente emanata. E' questa l'opinione di P. Ferrua, Il "giusto" processo, Zanichelli, 2012, p. 47 s. e di M. Taruffo, La semplice verità, cit., p. 97 s., 117 s.; Id., Contro la veriphobia, cit., p. 1003: poiché la giustizia di una condanna presuppone la verità dell'affermazione di colpevolezza, a quel giudizio retrospettivo di falsità dovrebbe necessariamente accompagnarsi un giudizio retrospettivo di ingiustizia della decisione (P. Ferrua, ivi, p. 50: «quando sopraggiunga la prova della falsità [delle premesse in fatto poste a fondamento della sentenza], non si dirà che la sentenza, giusta allora, è divenuta ingiusta oggi, allo stesso modo in cui nessuno direbbe che l'enunciato vero è divenuto falso»). Più in generale, la condanna dell'innocente sarebbe sempre ingiusta: anche quando le regole del (giusto) processo siano state correttamente rispettate, anche quando le norme di diritto sostanziale siano state 
6. Riassumendo, non c'è alcun valido motivo per considerare la verità del processo penale una verità minore, formale, convenzionale ecc. Non è un buon argomento la natura innegabilmente antiepistemica di molte delle regole del processo. Divieti probatori estrinseci e strettoie rituali rendono la ricerca giudiziale della verità senz'altro meno agevole della ricerca storica o scientifica: ma quando il giudice condanna, la verità che conduce l'imputato in carcere non è certamente una verità minore o deteriore - guai se lo fosse - rispetto a quella dello storico, del giornalista o dello scienziato. Non è un buon argomento neppure la natura fatalmente probabilistica dei giudizi di fatto che sorreggono l'affermazione di colpevolezza. Quando i dubbi che permangono sono unicamente di natura scettica, sarebbe irrazionale ritenere non accertato il fatto: e per quanto detto circa i rapporti tra verità e giustificazione, ritenere accertato un fatto significa ritenere vero l'enunciato che lo descrive. Le sentenze di condanna conformi al canone decisorio dell'assenza di ragionevoli dubbi vengono emanate quando le ragioni per ritenere falsa l'accusa hanno carattere esclusivamente logico, cioè non sono che l'astratta possibilità di una ragione. Tanto basta per attribuire razionalmente a simili verdetti - fedeli all'etimologia del vocabolo - la patente di affermazioni veritiere, senza ulteriori inutili specificazioni.

\section{REFERÊNCIAS}

AMODIO, Ennio. Verso una storia della giustizia penale in età moderna e contemporanea, em Criminalia, 2010.

BOGHOSSIAN, Paul. Paura di conoscere. Contro il relativismo e il costruttivismo, Roma: Carocci, 2006.

correttamente applicate, anche quando la condanna sia stata correttamente pronunciata oltre ogni ragionevole dubbio allo stato delle conoscenze e sulla base delle prove disponibili (queste le condizioni di giustizia della decisione - ulteriori rispetto alla verità dell'affermazione di colpevolezza - secondo gli autori citati). La giustizia della condanna dipenderebbe, insomma, anche da una condizione esterna al processo: da come sono andate effettivamente le cose e non da come era ragionevole ritenere che fossero andate nel momento in cui la sentenza è stata pronunciata; dalla verità dell'affermazione di responsabilità e non dalla sua giustificazione razionale. 
CAPRIOLI, Francesco. L'accertamento della responsabilità penale "oltre ogni ragionevole dubbio", em Riv. it. dir. proc. pen., 2009.

CAPRIOLI, Francesco. La scienza "cattiva maestra": le insidie della prova scientifica nel processo penale, em Cass. pen., 2008.

CARRARA, Francesco. Programma del corso di diritto criminale. Del giudizio criminale, con una selezione dagli Opuscoli di diritto criminale, Bologna: Il Mulino, 2004.

CAVALLONE, Bruno. In difesa della veriphobia (considerazioni amichevolmente polemiche su un libro recente di Michele Taruffo), em Riv. dir. proc., 2010.

COMANDUCCI, Paolo. La motivazione in fatto, em AA.VV. (org.), La conoscenza del fatto nel processo penale, Milano: Giuffré, 1992.

CORDERO, Franco. Prove illecite, em Tre studi sulle prove penali, Milano: Giuffrè, 1963.

D'AGOSTINI, Franca. Introduzione alla verità, Torino: Bollati Boringhieri, 2011.

DAMAŠKA, Mirjan. Il diritto delle prove alla deriva, Bologna: Il Mulino, 2003.

DAVIDSON, Donald. Sulla verità, Bari-Roma: Laterza, 2006.

DERSHOWITZ, Alan. Dubbi ragionevoli. Il sistema della giustizia penale e il caso O. J. Simpson, Milano: Giuffrè, 2007.

DUMMETT, Michael. Verità e passato, Milano: Raffaello Cortina Editore, 2006.

ENGEL, Pascal; RORTY, Richard. A cosa serve la verità, Bologna: Il Mulino, 2007.

FERRAJOLI, Luigi. Diritto e ragione. Teoria del garantismo penale, Bari-Roma: Laterza, 1991.

FERRUA, Paolo. Il giudizio penale: fatto e valore giuridico, in AA.VV. (org.), La prova nel dibattimento penale, Torino: Giappichelli, 2010.

FERRUA, Paolo. Contraddittorio e verità nel processo penale, in Studi sul processo penale, II, Anamorfosi del processo accusatorio, Torino: Giappichelli, 1992.

FERRUA, Paolo. Il “giusto” processo, Bologna: Zanichelli, 2012.

FESTA, Roberto. Induzione, probabilità e verisimilitudine, in G. Giorello, Introduzione alla filosofia della scienza, Milano: Bompiani, 1999.

IACONA, Andrea. L'argomentazione, Torino: Einaudi, 2005.

KOSTORIS, Roberto. Voce Giudizio (dir. proc. pen.), em Enc. giur. Treccani, vol. XV, Agg. 1997.

KÜNNE, Wolfgang. Conception of Truth, Oxford: Clarendon Press, 2003.

LOZZI, Gilberto. Lezioni di procedura penale, Torino: Giappichelli, 2011. 
LUCCHINI, Luigi. Elementi di procedura penale, Firenze: Barbéra Editore, 1920. MARCONI, Diego. Per la verità. Relativismo e filosofia, Torino: Einaudi, 2007. NAGEL, Thomas. L'ultima parola: contro il relativismo, Milano: Feltrinelli, 1999. NEGRI, Daniele. Fumus commissi delicti. La prova per le fattispecie cautelari, Torino: Giappichelli, 2004.

NOBILI, Massimo. Il principio del libero convincimento del giudice, Milano: Giuffré, 1974.

NOBILI, Massimo. L'immoralità necessaria. Citazioni e percorsi nei mondi della giustizia, Bologna: Il Mulino, 2009.

ORLANDI, Renzo. Verità, responsabilità e ravvedimento tra processo penale e pratiche di mediazione, em Corte Assise, 2011.

PEIRCE, Charles Sanders. Definitions of Truth, in Collected Papers, a cura di C. Hartshorne, P. Weiss e A. Burks, Cambridge: Belknap Press, vol. V, 1932.

PUTNAM, Hilary. Ragione, verità e storia, Milano: Il Saggiatore, 1981.

QUINE, Van Orman. Parola ed oggetto, Milano: Il Saggiatore, 1996.

RAMSEY, Frank Plumpton. Fatti e proposizioni, in I fondamenti della matematica e altri scritti di logica, Milano: Feltrinelli, 1964.

RORTY, Richard. La verità è una meta della ricerca?Donald Davidson contro Crispin Wright, in Verità e progresso. Scritti filosofici, Milano: Feltrinelli, 2003.

TARSKI, Alfred. La concezione semantica della verità e i fondamenti della semantica, in AA.VV., Semantica e filosofia del linguaggio, a cura di L. Linsky, Milano: Il Saggiatore, 1969.

TARUFFO, Michele. La prova dei fatti giuridici. Nozioni generali, Milano: Giuffré, 1992.

TARUFFO, Michele. Contro la veriphobia: osservazioni sparse in risposta a Bruno Cavallone, em Riv. dir. proc., 2010.

TARUFFO, Michele. La semplice verità, Bari-Roma: Laterza, 2009.

TUOZZI, Pasquale. Principii del procedimento penale italiano, Stabilimento Ripografico M. D’Auria, 1909.

UBERTIS, Giulio. Conoscenza fattuale e razionalità della decisione giudiziale, em Argomenti di procedura penale, Milano: Giuffré, 2002.

UBERTIS, Giulio. Fatto e valore nel sistema probatorio penale, Milano: Giuffré, 1979. 
VICOLI, Daniele. La "ragionevole durata" delle indagini, Torino: Giappichelli, 2012.

VOLPE, Giorgio. Teorie della verità, Milano: Guerini Scientifica, 2005.

WILLIAMS, Bernard. Truth and Truthfulness, Princeton: Princeton University Press, 2002.

\section{Dados do processo editorial}

(http://www.ibraspp.com.br/revista/index.php/RBDPP/about/editorialPolicies)

- Recebido em: 28/01/2016

- Versão final autor: 12/12/2016

- Autor convidado

http://www.ibraspp.com.br/revista/index.php/ RBDPP/about/editorialPolicies\#custom-1

\section{Equipe editorial envolvida}

- Editor-chefe: 1 (VGV)

- Editores-assistentes: 2 (BC e CRG)

\section{COMO CITAR ESTE ARTIGO:}

CAPRIOLI, Francesco. Verità e giustificazione nel processo penale. Revista Brasileira de Direito Processual Penal, Porto Alegre, vol. 3, n. 1, p. 317-342, jan./abr. 2017. https://doi.org/10.22197/rbdpp.v3i1.30

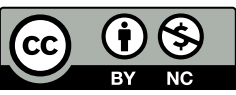

Esta obra está licenciada com uma Licença Creative Commons Atribuição-NãoComercial 4.0 Internacional. 